\section{Fadenförmige Wachstumsformen bei der Elektrokristallisation des Silbers}

Von Ludwig Graf und Wolfram Morgenstern

Institut für Metallphysik am Max-Planck-Institut für Metallforschung, Stuttgart

(Z. Naturforschg. 10a, 345-346 [1955]; eingeg. am 18. März 1955)

Bei der elektrolytischen Abscheidung von Silber aus wäßriger $\mathrm{AgNO}_{3}$-Lösung lassen sich unter gewissen Bedingungen fadenartige Kristallformen von wenigen $\mu$ Durchmesser feststellen. Für prinzipielle Untersuchungen des elektrolytischen Wachstumsmechanismus hat diese Wachstumsform bisher wenig Beachtung gefunden. Nur von Samarzew ${ }^{1}$ und Vahramian ${ }^{2}$ liegen Arbeiten über Silberfäden vor, die sich aber mehr mit den allgemeinen Entstehungsbedingungen und der Wachstumsgeschwindigkeit dieser Kristallform befassen. Dagegen konnte deren Struktur und Wachstumsmechanismus nicht befriedigend geklärt werden, da die verwendete Versuchsanordnung nur eine Betrachtung mit geringer Vergrößerung zuließ. Fis cher ${ }^{3}$ kam auf Grund der Beobachtungen von Samarzew und Vahramian zu der Auffassung, daß die Fäden einzelnen Lamellen (Wachstumsschichten) entsprechen, die isoliert in den Elektrolyten hineinwachsen und einen etwa kreisförmigen Querschnitt besitzen. An der krummen Fadenstirnfläche sollte dann ein ,normales' (statistisches) Wachstum im Sinne der Abscheidung fern vom Phasengleichgewicht nach Graf ${ }^{4}$ auftreten.

Eingehende Untersuchungen ${ }^{5}$ mit einer Versuchsanordnung, die die größtmögliche lichtoptische Auflösung bei unmittelbarer Betrachtung des Wachstums auszunutzen gestattete, sowie elektronenmikroskopische Aufnahmen der Silberfäden, zeigten nun

erstens, daß bei der fadenförmigen Kristallisationsart zwei wesentlich verschiedene Wachstumsformen auftreten, nämlich

a) eine verschlungene, nur auf der Kathodenfläche auswachsende ,Band'-Form, die offensichtlich ein monolamellares Wachstum mit statistischer Anlagerung ähnlich wie am Rande einer Wachstumslamelle darstellt;

b) eine mit konstantem Querschnitt in beliebiger Richtung in den Elektrolyten hineinwachsende gerade ,Nadel'-Form.

Zweitens konnte festgestellt werden, daß die ,Nadeln' allseitig von kristallographisch definierten Ebenen begrenzt sind. Sie besitzen einen rechteckigen Querschnitt und weisen ein lamellares Wachstum auf den Nadelstirnflächen auf.

Das Auftreten von Nadeln oder Bändern konnte nur in einem Strombereich von etwa $10^{-8}$ bis $10^{-6} \mathrm{Amp}$. beobachtet werden. Als Kathoden wurden die Quer-

${ }^{1}$ A. G. Samarzew, C. R. de l'Academie des Sic. USSR 2, 478 [1935].

2 A. T. Vahramian, J. phys. Chem. (russ.) 10, 443 [1937].

${ }^{3}$ H. Fischer, Elektrolytische Abscheidung und schnitte von in Araldit eingebetteten Platin- oder Silberdrähten mit 3 bis $150 \mu$ Durchmesser verwendet. Als Elektrolyt zeigte sich eine $0,1-n$. bis $3-n$. wäßrige $\mathrm{AgNO}_{3}$-Lösung, die man einige Wochen in geschlossenem Glasgefä $B$ altern lie $\beta$, geeignet. Beimengungen von $\mathrm{KNO}_{3}$ oder $\mathrm{KCl}$ änderten an der Wachstumsform nichts, während Zusätze von $\mathrm{KJ}, \mathrm{KBr}$ und $\mathrm{KCN}$ ein Nadelwachstum vollständig verhinderten. Die Nadeln entstanden entweder durch Abschnürung eines globulistisch auswachsenden Kriställchens bei Stromverringerung oder wuchsen bei Strömen kleiner als $10^{-6}$ Amp. direkt aus einer ungeordneten Silberabscheidung heraus.

Die Abmessung der Schmalseite (Abb. $2 *$ ) des rechteckigen Nadelquerschnitts betrug stets etwa 1 bis $2 \mu$ und war unabhängig vom Gesamtstrom, während die Abmessungen der Breitseite (Abb. 1 a und b) im Strombereich von $10^{-8}$ bis $10^{-6}$ Amp. von 2 bis auf etwa $20 \mu$ anstiegen. Die seitlichen Begrenzungen der Nadeln sind Würfelflächen; die pultförmige Stirnfläche (Abb .3) wird durch die $\{210\}$ - und die $\{230\}$-Ebene gebildet, in einigen seltenen Fällen auch durch zwei $\{210\}$-Ebenen.

Das lamellare Wachstum an der Nadelstirnfläche erfolgte bei breiteren Exemplaren auf beiden Stirnflächenebenen gleichzeitig durch Ausbreiten von Lamellen von den Schmalseiten zur Nadelspitze oder auch von dieser zu den Schmalseiten hin. Bei schmäleren Kristallen wurde die $\{230\}$-Ebene beim Wachstum nur durch den Rand der Lamelle auf der $\{210\}$-Ebene gebildet (Abb. 3). Hierbei erhielt man bei seitlicher Betrachtung der Nadel den Eindruck einer sich aneinanderreihenden Perlenkette (Abb. 2). Die Summe der Lamellenrandflächen auf der Nadelstirnfläche scheint unabhängig von der Nadelbreite stets etwa $1 / 5$ der gesamten Stirnfläche zu betragen.

In dem erwähnten Strombereich blieb infolge der Abhängigkeit der Nadelbreitseite vom Strom die Stromdichte, bezogen auf die Nadelstirnfläche, konstant und betrug etwa $2 \mathrm{Amp}$. $/ \mathrm{cm}^{2}$. Durch das ,Flächenverhältnis' von 1:5 kommt man so zu einer ebenfalls konstanten Stromdichte am ,normal' auswachsenden Lamellenrand (tatsächliche Wachstumsstelle) von $10 \mathrm{Amp} . \mathrm{cm}^{2}$. Dies würde einer Abscheidung von $10^{5}$ atomaren Schichten pro sec entsprechen und eine statistische Anlagerung wahrscheinlich machen. Beimengungen von Fremdstoffen änderten diese Stromdichten nur in kleinen Grenzen. Die Konstanz der Stromdichte auf einer lamellar auswachsenden Ebene bringt die Gesamtfläche dieser Ebene in Beziehung zum lamellaren Wachstumsmechanismus.

Unter den gleichen Bedingungen, unter denen ein Nadelwachstum auftrat, konnte auch ein monolamellares Bandwachstum beobachtet werden. Die eine Wachstumsart konnte unvermittelt in die andere umschlagen (Abb. 4). Die Bandbreite stieg mit dem Strom.

Elektrokristallisation von Metallen, Springer-Verlag Berlin-Heidelberg, 1954.

${ }^{4}$ L. Graf, Z. Metallkunde 45, 36 [1954].

${ }^{5}$ Die ausführliche Veröffentlichung erfolgt in der Z. Elektrochem.

* Abb. 1-6 auf Tafel S. 344 a. 
An der Stirnfläche des Bandes war das gleiche undefinierte ,Fließen' zu beobachten wie an einem sich über eine Ebene ausbreitenden Lamellenrand. Zufällig auftretende Verbreiterungen des Bandes führten zum Stillstand des Wachstums, sobald die Stromdichte auf der Stirnfläche unter den zur Anlagerung erforderlichen Wert sank. Erst durch erneute Keimbildung konnte dann das Band weiterwachsen, so daß Abstufungen auftraten (Abb. 4). Die Abbildungen, vor allem des verschlungenen Bandes (Abb. 5), vermitteln nicht den gleichen Eindruck wie die unmittelbare Betrachtung des Wachstums, da während der Belichtungs- zeit Vergröberungen auftraten. Das Bandwachstum scheint etwa der Fischerschen Auffassung vom fadenförmigen Wachstum des Silbers zu entsprechen.

Die lamellar wachsende Nadelstirnfläche stellt mit ihrer definierten Ebene den einzigen Ansatzpunkt für Untersuchungen des lamellaren Wachstumsmechanismus auf einer Ebene mit zeitlich konstanten Ausmessungen dar, da sich bei einem globulistisch auswachsenden Kristall die einzelnen lamellar wachsenden Ebenen gegenseitig beeinflussen.

Der Deutschen Forschungsgemeinschaft danken wir für die Bereitstellung von Apparaten.

\section{Wachstumslamellen auf Kupferkristallen}

Von L. Graf und L. R. Hell

Institut für Metallphysik am Max-Planck-Institut für Metallforschung, Stuttgart

(Z. Naturforschg. 10a, 346 [1955]; eingeg. am 28. März 1955)

In Fortsetzung früherer Untersuchungen über die Lamellenstrukturen, die sich auf den Oberflächen sauber erstarrter Metallschmelzen ausbilden ${ }^{1}$, wurde versucht festzustellen, auf welchen kristallographisch definierten Ebenen überhaupt derartige Lamellenstrukturen auftreten, wenn allen Ebenen möglichst gleiche Bedingungen zu ihrer Ausbildung geboten werden. Dies gelang durch Erstarrenlassen z. B. von Kupferschmelzen in einer etwas gegen die Horizontale geneigten Graphitrinne, wobei sich das tiefer liegende freie Ende des Schmelzguts unter dem Einfluß von Oberflächenspannung und Schwerkraft mit starker, angenähert kugeliger Krümmung ausbildete. Von diesem Ende aus erstarrte die Schmelze unter Schutzgas von Normaldruck in einem Längstemperaturgefälle einkristallin ohne Schrumpfungen. Die so erzeugten Einkristalle hatten eine hochglänzende Oberfläche mit farbig opaleszierenden Bereichen, die an dem gleichmäßig stark gekrümmten Ende der Einkristalle besonders regelmäßig ausgebildet waren und deutlich die Symmetrie der betreffenden Pole erkennen ließen (Abb. $1^{*}$ verzerrte, Abb. 2 unverzerrte Ausbildung um 〈111〉-Pole, Abb. 3 unverzerrte Ausbildung um einen $\langle 110\rangle$-Pol). Diese makroskopischen Erscheinungsbilder werden, wie eingehende Untersuchungen zeigten ${ }^{2}$, durch mindestens vier Lamellensysteme um kristallographisch verschiedene Zentren hervorgerufen. So erkennt man auf Abb. 4, die etwas schwächer belichtet ist als Abb. 2, als Lamellenzentrum der inneren Scheibe den $\langle 111\rangle$-Pol, und der hell reflektierende Mittelpunkt entspricht der reell ausgebildeten $\{111\}$-Ebene. Dagegen gehören die sich mit geringem Zwischenraum an die Scheibe anschließenden ,,Strahlen“ anderen Lamel-

${ }^{1}$ L. Graf, Z. Elektrochem. 48, 181 [1942]; Z. Metallkunde 42, 336 u. 401 [1951]; Z. Metallkunde 45, 36 [1954].

* Abb. 1-5 auf Tafel S. 344 b.

${ }^{2}$ L. R. Hell, Diplomarbeit T. H. Stuttgart 1954.

${ }^{3}$ J.A. A. Leroux u. E. Raub, Z. anorg. allg. Chem. lenzentren an, und zwar die drei längeren Strahlen, die sich in Richtung auf die $\langle 110\rangle$-Pole erstrecken, dem Lamellenzentrum um diese Pole, und die jeweils dazwischen liegenden drei kürzeren Strahlenpaare Lamellenzentren um die $\langle 241\rangle$-Pole. Außerdem liegen noch weitere Lamellenzentren in den $\langle 8,16,1\rangle$-Polen, wobei sich jedoch die entsprechenden Lamellenstrukturen nur auf den $\langle 2 \overline{1} 0\rangle$-Zonen zwischen den $\langle 241\rangle$ und $\langle 24 \overline{1}\rangle$-Polen stärker ausbilden. Desgleichen treten bei den Lamellensystemen um die $\langle 241\rangle$-Pole Lamellenstrukturen nur auf den $\langle 4 \overline{3} 4\rangle$-Zonen zwischen den $\langle 241\rangle$ - und $\langle 142\rangle$-Polen auf. Unmittelbar um die $\langle 110\rangle$-Pole ist die Lamellenstruktur sehr schwach ausgebildet, wie man an dem inneren dunklen Ring erkennt; außerdem ist die $\{110\}$-Ebene am Pol nur wenig entwickelt. Auffallend ist, daß um keine anderen nieder indizierten Pole Lamellensysteme auftreten, insbesondere nicht um die $\langle 100\rangle$-Pole, obwohl die Würfelebenen reell und von gleicher Größe wie die $\{111\}$ Ebenen erscheinen.

Wie durch sorgfältige Untersuchungen festgestellt worden ist ${ }^{2}$, handelt es sich bei diesen Lamellen zweifellos um Wachstumsstrukturen. Bekanntlich entstehen auch beim Abbau der Kristalle ähnliche Lamellenstrukturen, wie z. B. bei Kupfer, Silber und Platin beobachtet worden ist ${ }^{3}$. Ihre Entstehung ist jedoch an eine Einwirkung von Sauerstoff gebunden, dessen Einfluß bei unseren Untersuchungen zuverlässig ausgeschlossen worden ist. Im Gegensatz zum Aufbau bilden sich beim Abbau auch um die Würfel-Pole Lamellenterrassen aus, was man unmittelbar als Kriterium für das Vorliegen einer Abbau-Struktur ansehen kann. Dieses unterschiedliche Verhalten bestimmter Ebenen hinsichtlich der Ausbildung von Lamellenterrassen ist z. Z. noch ungeklärt und Gegenstand weiterer Untersuchungen. Eine ausführliche Veröffentlichung erscheint demnächst in der Zeitschrift für Kristallographie.

Der Deutschen Forschungsgemeinschaft haben wir für die Bereitstellung von Apparaten zu danken.

188, 208 [1930]; B. Chalmers, R. King u. R. Shuttleworth, Proc. Roy. Soc. A 193, 465 [1948]; E. Menzel, Naturwiss. 37, 166 [1950]; Z. Phys. 132, 508 [1952]; L. Graf, Z. Elektrochem. 48, 181 [1942] sowie unveröffentlichte Beobachtungen an Silber-Einkristallkugeln. 